\title{
Level set and Thresholding for Brain Tumor Segmentation
}

\author{
N. S. Zulpe, COCSIT, Latur, V. P. Pawar, and SRTMU, Nanded
}

\begin{abstract}
Internal structure of the human body obtained by different modalities such as Computed Tomography (CT), Magnetic Resonance Imaging (MRI), etc. Images from these modalities are generally used for the diagnosis purpose. In the Brain MRI image, the tumor part can be seen clearly but the physician need the accurate size and the correct measurement for the treatment. This requires to segment the tumor part from the given Brain MRI image and quantify it. We have used Brain tumor (Metastatic bronchogenic carcinoma) MRI scans of 42-year-old woman from the Whole Brain Atlas (WBA) for the experiment; in this paper, we presented the basic level set method to catch the boundaries of the tumor area in 2D MRI images. After catching the accurate boundaries, we applied the inverse threshold and binary mask to segment the exact tumor region from the given different MRI brain scans. At last, we applied some of the statistical function on the region of interest (ROI) to quantify the tumor region.
\end{abstract}

Index Terms-CT, MRI, WBA, level set, ROI.

\section{INTRODUCTION}

Image segmentation is a broad and active field in medical imaging. Its purpose is to divide an image into regions, which are meaningful for a particular task. Various methods and approaches are used; the choice of a particular method depends on the characteristics of the problem to be solved and its place in a wider image analysis strategy. Segmentation is an essential step prior to the description, recognition, or classification of an image or its constituents. There are two major approaches - region-based methods, in which similarities are detected and boundary-based methods, in which discontinuities (edges) are detected and linked to form boundaries around regions [1].

In this paper, we have used the level set method to find out the tumor part from the given MRI images. The level set method gradually develop a contour (in two dimensions) or a surface (in three dimensions) implicitly by manipulating a higher dimensional function, called the level set function $\phi(X, t)$. The evolving contour or surface can be extracted from the zero level set $\Gamma(X, t)=\{\phi(X, t)=0\}$.

The advantage of using this method is that topological changes such as merging and splitting of the contour or surface are catered for implicitly, the level set method, since its introduction by Osher and Sethian [2], has seen widespread application in image processing.

Manuscript received December 21, 2011; revised February 27, 2012.

Nitish Zulpe is with the College of Computer Science and Information Technology, Latur, Maharashtra (e-mail: nitish.zulpe@gmail.com)

Vrushsen Pawar is with department of computational science, SRTM University Nanded, Maharashtra (e-mail: vrushvijay@yahoo.co.in)
Kass et al. [3] introduced segmentation using active contours model (Snakes). The idea behind active contours, or deformable models, for image segmentation is quite simple. The user specifies an initial guess for the contour, which is then moved by image driven forces to the boundaries of the desired objects. In such models, two types of forces are considered - the internal forces, defined within the curve, are designed to keep the model smooth during the deformation process, while the external forces, which are computed from the underlying image data, are defined to move the model toward an object boundary or other desired features within the image. One way of describing this curve is by using an explicit parametric form, which is the approach used in snakes. This causes difficulties when the curves have to undergo splitting or merging, during their evolution to the desired shape. To address this difficulty, the implicit active contour approach, instead of explicitly following the moving interface itself, takes the original interface and embeds it in higher dimensional scalar function, defined over the entire image. The use of level set method has provided more flexibility and convenience in the implementation of active contours. Active contours [4] have been introduced for segmenting deformable objects, by defining the internal and external functional and minimizing the functional whose local maximum is located at the object boundary. But, active contours are relatively noise sensitive, and their results depend on the initialization and they are not sufficiently topologically adaptive. Chunming Li. et. al. [5] proposed a new region-based active contour model in a variational level set formulation. They first defined a region-scalable fitting $(R S F)$ energy functional in terms of a contour and two fitting functions that locally approximate the image intensities on the two sides of the contour. The optimal fitting functions are shown to be the averages of local intensities on the two sides of the contour. The regionscalability of the RSF energy is due to the kernel function with a scale parameter, which allows the use of intensity information in regions at a controllable scale, from small neighbor-hoods to the entire domain. This energy is then incorporated into a variational level set formulation with a level set regularization term. In the resulting curve evolution that minimizes the associated energy functional, intensity information in local regions at a certain scale is used to compute the two fitting functions and, thus, guide the motion of the contour toward the object boundaries.

Fuping Zhu and Jie Tian [6] to segment an object from a medical image. They used the idea of the fast marching and level set technique. First, the rough boundaries of interested object are extracted by fast marching method. The extracted edges are regarded as an initialization of level set method. They modified the traditional fast marching method in order to escape the error results. Then, the level set method is used to finely tune and smooth the contour acquired by fast 
marching method.

Chunming Li. et al. [7] proposed another novel regionbased method for image segmentation. From a generally accepted model of images with intensity in homogeneities, they derived a local intensity clustering property, and therefore defined a local clustering criterion function for the intensities in a neighbor-hood of each point. This local clustering criterion is integrated over the neighborhood center to define an energy functional, which is converted to a level set formulation. Minimization of this energy is achieved by an interleaved process of level set evolution and estimation of the bias field. As an important application, our method can be used for segmentation and bias correction of magnetic resonance (MR) images. M. Airouche et.al. [10] explore image segmentation using active contours model to detect oil spills. A partial differential equation based level set method, which represents the spill surface as an implicit propagation interface, is used. Starting from an initial estimation with priori information, the level set method creates a set of speed functions to detect the position of the propagation interface. Specifically, the image intensity gradient and the curvature are utilized together to determine the speed and direction of the propagation. This allows the front interface to propagate naturally with topological changes, significant protrusions and narrow regions, giving rise to stable and smooth boundaries that discriminate oil spills from the surrounding water.

Segmentation techniques can be classified as either contextual or non-contextual. Non-contextual techniques ignore the relationships that exist between features in an image; pixels are simply grouped together on the basis of some global attribute, such as gray level. Intensity-based thresholding, where each pixel is assigned to a particular region based on its gray value, is a non-contextual technique. Contextual techniques additionally exploit the relationships between image features. Thresholding is one of the segmentation techniques, which can be used for MRI brain tumor segmentation [1]. According to intensity/brightness is a simple technique for images, which contain solid objects on a background of different, but uniform, brightness. Each pixel is compared to the threshold: if its value is higher than the threshold, the pixel is considered to be "foreground" and is set to white, and if it is less than or equal to the threshold it is considered "background" and set to black. Chowdhury et.al. [11] introduced a new image thresholding method based on the divergence function. In this method, the objective function is constructed using the divergence function between the classes, the object and the background .The required threshold is found where this divergence function shows a global minimum. In contourbased methods, Lefohn et al. [12] have proposed a semiautomatic method for tumor segmentation using level sets. The user selects the tumor region, to initialize a first segmentation. Based on a visual inspection of the results, he tunes the level set parameters and the segmentation process is repeated.

\section{Methods AND Materials}

\section{A. Magnetic Resonance Imaging}

A magnetic resonance imaging instrument (MRI Scanner) uses powerful magnets to polarize and excite hydrogen nuclei i.e. proton in water molecules in human tissue, producing a detectable signal which is spatially encoded, resulting in images of the body. MRI uses three electromagnetic fields

1) A very strong static magnetic field to polarize the hydrogen nuclei, called the static field.

2) A weaker time varying field(s) for spatial encoding called the gradient field.

3) A weak radio frequency field for manipulation of hydrogen nuclei to produce measurable signals collected through RF antenna.

We have used Brain tumor (Metastatic bronchogenic carcinoma) MRI images of 42 year old woman with a long history of tobacco use began having headaches one month before these images were obtained. Brain images show a large mass with surrounding edema, and compression of adjacent midbrain structures. The MR demonstrates the tumor as an area of high signal intensity on proton density (PD) and T2-weighted (T2) images in a large left temporal region, these images taken from Whole Brain Atlas (WBA).

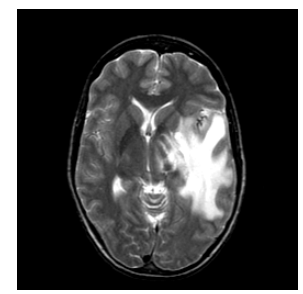

Fig. 1. Tumor affected MRI brain image.

\section{B. Level-set Method}

Level-sets methods [2] rely on partial differential equations (PDEs) to model deforming isosurfaces. Levelsets methods rely on two central embeddings; first the embedding of the interface as the zero level set of a higher dimensional function, and second, the embedding (or extension) of the interface's velocity to this higher dimensional level set function. The evolution of the contour or surface is governed by a level set equation. The solution tended to by this partial differential equation is computed iteratively by updating $\phi$ at each time interval, the general form of the level set equation is shown below.

$$
\frac{\partial \phi}{\partial t}=-|\nabla \phi| \cdot F
$$

In the above level set equation $F$ is the velocity term that describes the level set evolution. By manipulating $F$, we can guide the level set to different areas or shapes, given a particular initialization of the level set function. In [7] $F$ is dependent on data and curvature functions only (with a weighting parameter between the two) for the purposes of image segmentation. Therefore, we adopt the same methodology making the level set equation take the form

$$
\frac{\partial \phi}{\partial t}=-|\nabla \phi| \cdot\left[\alpha D(I)+(1-\alpha) \nabla \cdot \frac{\nabla \phi}{|\nabla \phi|}\right]
$$

where the data function $D(I)$ tends the solution towards targeted features, and the mean curvature term $\nabla \cdot \nabla \phi /|\nabla \phi|$ 
keeps the level set function smooth. Weighting between these two is $\alpha \in[0,1]$ a free parameter that is set beforehand to control how smooth the contour should be.

The data function $D(I)$ acts as the principal 'force' that drives the segmentation. By making $D$ positive in desired regions or negative in undesired regions, the model will tend towards the segmentation sought after. A simple speed function that fulfills this purpose, used by Lefohn, Whitaker and Cates in $[7,8]$, is given by

$$
D(I)=\epsilon-|I-T|
$$

Here $T$ describes the central intensity value of the region to be segmented, and $\epsilon$ describes the intensity deviation around $T$ that is part of the desired segmentation. Three user parameters that need to be specified for segmentation are $T, \epsilon$ and $\alpha$. An initial mask for the level set function is also required, which may take the form of a square in two dimensions, or any other arbitrary closed shape. The level set iteration can be terminated once $\phi$ has converged, or after a certain number of iterations.

\section{Signed Distance Transform}

A distance transform assigns a value for every pixel within a binary image containing one or more objects a value which represents the minimum distance from that pixel to the closest pixel on the boundary of the object(s). The Distance function $D(r, S)=\min |r-S|$ for the set $S$ mathematically represented as

$$
D(r, S)=\min |r-S| \quad \text { for all } r \in \mathbb{R}^{3}
$$

A signed distance transform assigns the sign of the distance value as positive for those pixels outside the object, and negative for those inside it. This is the sign convention that will be followed in the implementation, however the opposite sign convention could also be used. Signed distance transforms are required to initialize $\phi$ and to reinitialize it every certain number of iterations. Computation of the initialization of $\phi$ is required before iteration of the level set equation can take place, and this will typically be a signed distance transform of an initial mask. Therefore, the level set segmentation filter requires two images: an initial mask (which indicates targeted regions) and a feature image (which is the image to be segmented). In this paper, we have specified 10 arbitrary seed points to produce the initial contour with time step of $0.05 \mathrm{sec}$ and smooth curvature of 0.05 and the attractive force of 20. After 203 iterations, we got the correct tumor segmented image.
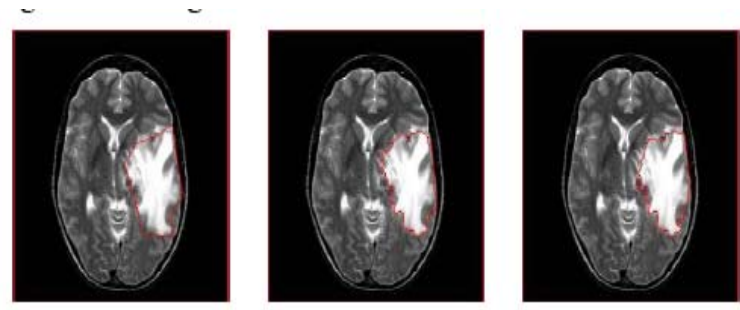

(a) Initial contour (b) 55 Iterations (c) 112 Iterations
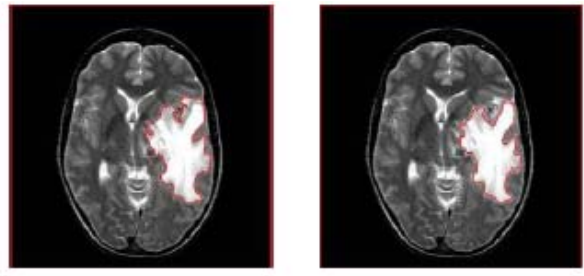

(d) 157 Iterations (e) 203 Iterations

Fig. 2. (a) Initial contour, (b-e) level set contour reaching the tumor region.

\section{Thresholding}

We have used the Inverse Standard Deviation (ISD) thresholding on the final contour of level set segmentation, which will calculate standard deviation (st.dev) of pixel intensities and other statistics. Then it will thresholds an image using the number of standard deviations and/or values outside the range. The thresholding can be done on the whole image. The image intensity values outside the threshold range are kept, and the ones in the threshold are set to the values outside.

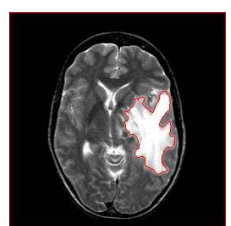

(a)

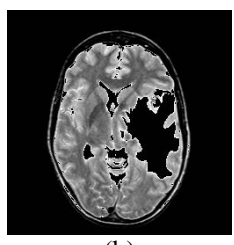

(b)
Fig. 3. (a) Segmented image, (b) Inverse thrsholded image

\section{E. Binarization and Masking}

Image binarization converts an image of up to 256 gray levels to a black and white image. Frequently, binarization is used as a pre-processor before OCR. In fact, most OCR packages on the market work only on bi-level (black \& white) images. The simplest way to use image binarization is to choose a threshold value, and classify all pixels with values above this threshold as white, and all other pixels as black. The problem then is how to select the correct threshold. In many cases, finding one threshold compatible to the entire image is very difficult, and in many cases even impossible. Therefore, adaptive image binarization is needed where an optimal threshold is chosen for each image area.

1) For the equalized image the pixels are represented in a 0 to 255 gray level intensity.

2) As the process is to extract the affected region or the accumulated region, a 2-level image representation would be sufficient for better computation.

3) For the binarization of equalized image a thresholding method is used as illustrated :

4) Binarized Image bi, $\mathrm{j}=255$ if e( $\mathrm{i}, \mathrm{j})>T$ else bi, $\mathrm{j}=0$ where $e(i, j)$ is the equalized MRI image and $T$ is threshold derived for the equalized image.

Then we define an ROI by creating a binary mask, which is a binary image that is the same size as the image you want to process with pixels that define the ROI set to 1 and all other pixels set to 0 . 


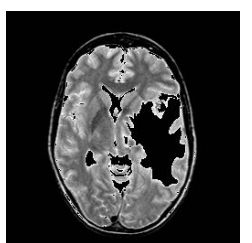

(a)

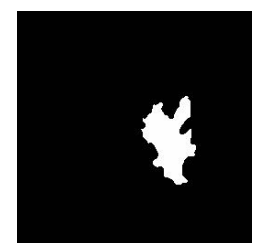

(b)
Fig. 4. (a) Inverse thrsholded image, (b) Binary mask segmented image.

\section{RESULTS}

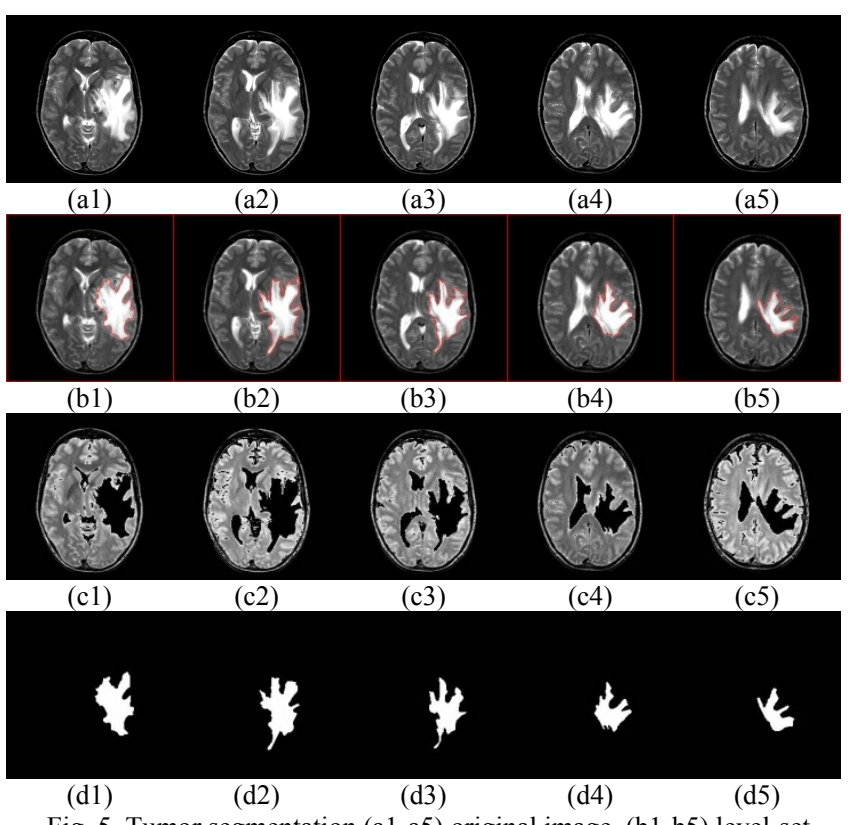

Fig. 5. Tumor segmentation (a1-a5) original image, (b1-b5) level-set segmented image, (c1-c5) Inverse thresholded image, (d1-d5) final segmentation.

table i: Measurement of Area, Perimeter, Geometric Center and CENTER OF MASS.

\begin{tabular}{ccccc}
\hline $\begin{array}{c}\text { ROI. } \\
\text { No }\end{array}$ & $\begin{array}{c}\text { Area } \\
\text { (Millimeters`2) }\end{array}$ & $\begin{array}{c}\text { Perimeter } \\
(\mathbf{m m})\end{array}$ & $\begin{array}{c}\text { Geometric } \\
\text { center } \\
\boldsymbol{x} / \boldsymbol{y} \text { in } \mathbf{m m}\end{array}$ & $\begin{array}{c}\text { Center } \\
\text { of Mass } \\
\boldsymbol{x} / \boldsymbol{y} \text { in } \\
\mathbf{m m}\end{array}$ \\
\hline ROI1 & 3263 & 347.26 & $166 / 144$ & $\begin{array}{c}166.34 / \\
140.11\end{array}$ \\
\hline ROI2 & 3331 & 416.91 & $163 / 147$ & $\begin{array}{c}163.26 / \\
147.75\end{array}$ \\
\hline ROI3 & 2621 & 402.90 & $161 / 150$ & $\begin{array}{c}161.19 / \\
150.09\end{array}$ \\
\hline ROI4 & 1939 & 298.44 & $159 / 152$ & $\begin{array}{c}158.84 / \\
152.33\end{array}$ \\
\hline ROI5 & 1612 & 267.48 & $155 / 155$ & $\begin{array}{c}155.42 / \\
155.66\end{array}$ \\
\hline \hline
\end{tabular}

TABLE 2: MEASUREMENT OF Principal AXIs, ECCENTRICITY, MaJor AND MINOR AXIS.

\begin{tabular}{ccccc}
\hline \hline $\begin{array}{c}\text { ROI. } \\
\text { No }\end{array}$ & $\begin{array}{c}\text { Principal Axis } \\
\text { (Degree) }\end{array}$ & Eccentricity & $\begin{array}{c}\text { Major } \\
\text { axis } \\
\text { length } \\
(\boldsymbol{m m})\end{array}$ & $\begin{array}{c}\text { Minor axis } \\
\text { length }(\boldsymbol{m m})\end{array}$ \\
\hline ROI1 & 83.1317 & 0.8021 & 83.4114 & 49.8083 \\
\hline ROI2 & -87.3477 & 0.8428 & 88.7696 & 47.7772 \\
\hline ROI3 & -88.2832 & 0.8067 & 75.1471 & 44.4084 \\
\hline ROI4 & 75.2649 & 0.5785 & 55.0152 & 44.8751 \\
\hline ROI5 & 58.5912 & 0.805 & 58.8187 & 34.8947 \\
\hline \hline
\end{tabular}

TABLE III: MEAsurement OF St.DeV, Median AND Mode InTENSITY AND MODE COUNT

\begin{tabular}{ccccc}
\hline $\begin{array}{c}\text { ROI. } \\
\text { No }\end{array}$ & $\begin{array}{c}\text { St. Dev of } \\
\text { Intensity }\end{array}$ & $\begin{array}{c}\text { Median } \\
\text { Intensity }\end{array}$ & $\begin{array}{c}\text { Mode } \\
\text { Intensity }\end{array}$ & $\begin{array}{c}\text { Mode } \\
\text { Count }\end{array}$ \\
\hline ROI1 & 54.22 & 254 & 254 & 1878 \\
\hline ROI2 & 60.54 & 254 & 254 & 1789 \\
\hline ROI3 & 65.97 & 254 & 254 & 1283 \\
\hline ROI4 & 64.7052 & 254 & 254 & 806 \\
\hline ROI5 & 68.1016 & 254 & 254 & 609 \\
\hline \hline
\end{tabular}

\section{CONCLUSION}

We used basic level set and inverse thresholding method to segment the tumor from the Metastatic bronchogenic carcinoma) MRI scans of 42-year-old woman from the Whole Brain Atlas (WBA). In this paper, we try to find out the contour around the tumor area in 2D MRI images. To specify initial contour we specified the arbitrary ten seed points around tumor, after final iteration, we applied the inverse threshold and binary mask to segment the exact tumor region from the given different MRI brain scans. At last, we applied some of the statistical function on the region of interest (ROI) to quantify the tumor region. The given method we presented is semiautomatic in future we try to convert it into fully automatic segmentation method, which will help physician for the better treatment.

\section{REFERENCES}

[1] G. Dougherty, Digital Image Processing for Medical Applications, 1st ed. CAMBRIDGE UNIVERSITY PRESS, Appendix B-4, pp. 309-310. 2009.

[2] S. Osher and J. A. Sethian, "Fronts propagating with curvature dependent speed: algorithms based on hamilton-jacobi formulations," Journal of Computational Physics, 79:12-49, 1988.

[3] M. Kass, A. Witkin, and T. Terzopoulous, "Snakes: Active contour models," International Journal of Computer Vision, pages 321-331, 1988.

[4] T. McInerney and D. Terzopoulos, "Deformable models in medical image analysis: a survey," Medical Image Analysis, 91-108, 1996.

[5] C. Li, C. Yen Kao, C. John Gore, and Z. Ding, "Minimization of Region-Scalable Fitting Energy for Image Segmentation," IEEE Transactions on Image Processing, Vol. 17, No. 10, October 2008.

[6] F. P. Zhu and J. Tian, "Modified fast marching and level set method for medical image segmentation," Journal of X-Ray Science and Technology 11 (2003) 193-204 193, IOS Press.2003.

[7] C. Li, R. Huang, Z. Ding, J. Chris Gatenby, N. Dimitris Metaxas, "A Level Set Method for Image Segmentation in the Presence of Intensity Inhomogeneities With Application to MRI," IEEE Transactions on Image Processing, Vol. 20, No. 7, July 2011.

[8] E. Aaron Lefohn, M. Joe Kniss, D. Charles. Hansen, and T. Ross Whitaker, "A streaming narrow-band algorithm: Interactive computation and visualization of level sets," IEEE Transactions on Visualization and Computer Graphics, 10:422 -433, 2004.

[9] J. E. Cates, A. E. Lefohn, and R. T. Whitaker, "GIST: an interactive, GPU-based level set segmentation tool for 3D medical images," Medical Image Analysis, 8(3):217-231, 2004.

[10] M. Airouche(1), L. Bentabet(2), and M. Zelmat (1), "Image Segmentation Using Active Contour Model and Level Set Method Applied to Detect Oil Spills," in Proc. World Congress on Engineering 2009, London, July 1 - 3, 2009.

[11] Chowdhury et.al. "Image thresholding techniques," in proc. IEEE Pacific Rim Conference on Communications, Computers, and Signal Processing, 17-19 May 1995, Page(s):585 - 589.

[12] A. Lefohn, J. Cates, and R. Whitaker, "Interactive, GPU-based level sets for 3D brain tumor segmentation," Technical Report, University of Utah, April 2003. 
[13] S. Osher and A. James Sethian, "Fronts propagating with curvature dependent speed: algorithms based on hamilton-jacobi formulations," Journal of Computational Physics, 79:12-49, 1988.

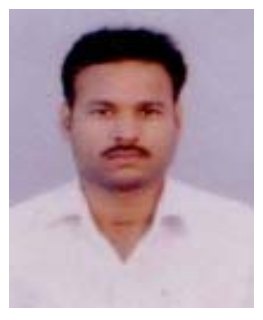

Nitish S Zulpe received the MCS degree from SRTM University, Nanded in the year 2004. He received the M.Phil. Degree in Computer Science from Y.C.M.O. University, Nashik in the year 2009. He is currently working as lecturer in the College of Computer Science and Information Technology, Latur, Maharastra. He is leading to $\mathrm{PhD}$ degree in University of Pune.

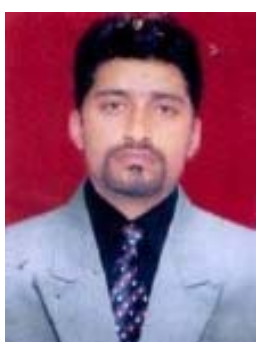

Vrushsen Pawar received MS, Ph.D.(Computer) Degree from Dept .CS \& IT, Dr.B.A.M. University \& PDF from ES, University of Cambridge, UK. Also Received MCA (SMU), MBA (VMU) degrees respectively. He has received prestigious fellowship from DST, UGRF (UGC), Sakaal foundation, ES London, ABC (USA) etc. He has published 90 and more research papers in reputed national international Journals \& conferences. He has recognize Ph.D Guide from University of Pune, SRTM University \& Sighaniya University (India). He is senior IEEE member and other reputed society member. Currently working as a Asso.Professor in CS Dept.SRTMU, Nanded 